\title{
Implementation of UV-photodissociation on a benchtop Q Exactive mass spectrometer and its application to phospho-proteomics
}

\author{
Kyle L. Fort1,2, Andrey Dyachenko1,2, Clement M. Potel1,2, Eleonora Corradini1,2, Fabio Marino1,2, \\ Arjan Barendregt ${ }^{1,2}$, Alexander A Makarov ${ }^{1,3}$, Richard A. Scheltema ${ }^{1,2}$, Albert J.R. Heck ${ }^{1,2, *}$ \\ *Corresponding Author
}

\section{Affiliation:}

1. Biomolecular Mass Spectrometry and Proteomics, Bijvoet Center for Biomolecular Research and Utrecht Institute of Pharmaceutical Sciences, Utrecht University, Utrecht, The Netherlands.

2. Netherlands Proteomics Center, Utrecht, The Netherlands.

3. Thermo Fisher Scientific (Bremen), Bremen, Germany.

\section{Contents:}

Figure S-1. Instrument performance at different modes of operation

Figure S-2. Database scores for HeLa digest before and after modification at various NCE values.

Figure S-3. Representation of electrical potentials used within HCD and UVPD mode of operation.

Figure S-4. Graphical depiction of the timescale of ion transport, activation, and mass analysis with both UVPD and HCD methods.

Figure S-5. Comparison of identified phosphopeptide ion with HCD and UVPD with $3 \mathrm{~mJ}$ (top) and 5mJ.

Figure S-6. Presence of UVPD specific fragments, where higher counts for a fragmentation method implies that that particular ion is more frequent for that method.

Table S-1:.LC gradients for the various samples analyzed by LC-MS.

Table S-2. Phospho peptide library.

Table S-3. Raw files correlated to Figures. 


\section{Effect of IonGun mode triggering and removal of the charge detector}

Temporal overlap of the ion packets and the photons is achieved by generating a pulse of the DC axial electric field within the HCD-cell with an amplitude of $-100 \mathrm{~V}$ for $1 \mathrm{~ms}$. This effectively confines the ion packet to the rear of the HCD-cell while triggering photon emission from the laser. Spatial overlap is achieved by replacing the in-line charge detector with a quartz viewport to allow the UV photons to enter the HCD-cell. The pulse introduced within the HCD-cell increases the magnitude of the axial electric field that ions are subjected to, potentially altering the internal energy distribution of the ions to a point where supplemental activation may be introduced. The number of PSMs in normal mode shows that the maximum performance is achieved at 24-26 NCE (Supplemental Figure 1a, blue). In the "IonGun" mode (Supplemental Figure 1a, green), the number of PSMs is almost identical at all tested NCE values to the normal mode of operation, indicating that indeed no supplemental activation is occurring. This is in line with expectations because voltage gradient along the axis of the HCD cell is about 2 orders of magnitude lower than the pulse applied to remote axial field electrodes and therefore ions entering along the axis cannot experience more than $1 \mathrm{~V}$ total acceleration or axial electric field of more than $0.01 \mathrm{~V} \mathrm{~mm}^{-1}$.

The removal of the charge detector increases the gas conductance out of the HCD-cell, affecting local pressure levels and potentially altering the fragmentation efficiency and/or decrease the ion transport efficiency. To explore these effects, the charge detector was removed from the HCD-cell and the tryptic HeLa analysis was repeated for the same range of NCE values. Unlike the data recorded with inclusion of the charge detector, a lower maximum number of PSMs was achieved at 20 NCE (Supplemental Figure 1a, red). The reduced performance upon removal of the charge detector increases with increasing NCE and maximizes at a 44\% reduction in PSMs at a NCE of 34. It is not immediately clear if this decrease in performance is caused by a reduction in fragmentation efficiency, a reduction in ion transport efficiency, or a combination of both. Comparison of peptide identification scores, which increases when more information rich fragment ions become available and reflects the quality of the $\mathrm{MS}^{2}$ spectrum, before and after the removal of the charge detector shows that the scores are largely identical for NCE values of 20 to 26 (Supplemental Figure 2). At the higher NCE values of 28-34, the scores show a small decrease. This is however not reflective of the $>40 \%$ decrease in PSMs observed at a NCE of 34, suggesting that ion transport efficiency, and not fragmentation efficiency, is the causative factor. Radial focusing employed in the HCD-cell for trapping ions depends on the momentum dampening of the ion within the device. When the translational energy of the ion prior to a collision event is increased, the momentum of the resulting fragments also increases. The reduced number density of the collision gas owing to the increased conductance upon modification, combined with the increased energy upon a scattering event at higher NCEs, can diminish the extent of radial confinement and lower ion transport efficiency. This effect can be counteracted by increasing the gas flow rate into the HCD-cell, causing the local number density of this region to increase. While the local pressure within the HCD-cell is not measureable, the flow rate directly affects the pressure within the Orbitrap mass analyzer, termed UHV. At the standard flow rate, with the removal of the charge detector, the UHV was $\sim 9.4 \mathrm{E}-11$ mBar. The flow rate into the HCD-cell was increased until the UHV was doubled to a value of $\sim 1.9 \mathrm{E}-10 \mathrm{mBar}$. Data collected at this higher gas flow rate (Supplemental Figure 1a, orange) shows that the general trend in PSMs and instrument performance is restored across the entire range of NCE values.

Generation of an external laser trigger: 
The utilized laser offers the option to operate in "continuous" mode or, via an external trigger, in synchronized pulse mode. Continuous mode fires the laser at a set frequency that is independent of the ion inject events occurring in the mass spectrometer. As ions reside in the HCD-cell for $\sim 2-10$ $\mathrm{ms}$ the maximum number of pulses one ion packet may be irradiated by is twice at a repetition rate of $200 \mathrm{~Hz}$. One potential drawback of the continuous mode is an increase in the background ion levels (both for full and fragmentation scans) due to the interactions of the laser beam with the Ctrap assembly. For the synchronized triggering, two approaches, termed "Ion injection" and “ IonGun" , were implemented to trigger the laser. During "Ion injection" , the delay generator is triggered by the opening of the split lens, which occurs during ion injection into the C-trap. As the opening of the split lens occurs for MS ${ }^{1}$ and MS ${ }^{2}$ scans, the downside of this method is that the trigger fires the laser during both events. The "IonGun" method utilizes an additional pulse of the HCD axial electric DC field (pulse of -100V for $1 \mathrm{~ms}$, see Supplemental Figure 3), which occurs only during a fragmentation scans. A potential drawback is that the increased potential drop across the HCD-cell increases the magnitude of the electric field within this region, potentially increasing collisional activation of the ions in the ion packet prior to UV irradiation. The extent of this additional collisional activation on the fragmentation of precursor ions is further addressed in the Results and Discussion.

\section{Data analysis}

All data was analyzed with Proteome DiscovererTM version 2.0 (Thermo Fisher Scientific), which handles centroiding, deisotoping, and precursor determination for all collected MS ${ }^{2}$ scans. ${ }^{1}$ For peptide identification, SEQUEST HT was utilized. ${ }^{2}$ This search algorithm supports equal score weighting of all fragment ion types required to support UVPD. ${ }^{3-5}$ Tandem mass spectra were searched against the International Protein Index human database (version 3.68; 87,061 entries), insilico digested by Trypsin with a maximum of 2 missed cleavages. The precursor and fragment mass tolerances were maintained at $20 \mathrm{ppm}$ and $0.05 \mathrm{Da}$, respectively. Oxidation of methionine was set as dynamic modification for the HeLa digest analyses, while phosphorylation of STY were added for the phospho-enriched analyses. A static modification of carbamidomethyl was used for all cysteines. Percolator with a false discovery rate (FDR) target of 0.05 and 0.01 , for relaxed and strict, respectively, was used. PhosphoRS ${ }^{6}$ was added for the phospho-enriched and synthetic phosphopeptide mass spectra and had a confidence score cutoff of 75\%. Additional analysis was performed with in-house tooling software capable of extracting meta-data from the raw data files based on MSFileReader (Thermo Fisher Scientific) and with the R scripting and statistical environment ${ }^{7}$ using ggplot. ${ }^{8}$ All the mass spectrometry-based proteomics data have been deposited to the ProteomeXchange Consortium via the PRIDE partner repository with the dataset identifier PXD003109 (http://www.ebi.ac.uk/pride; username: reviewer89560@ebi.ac.uk; password: fntTapJX). 


\section{Production of specific fragment ions}

To further investigate the differences between ions produced with each fragmentation method, details can be extracted from the fragmentation scans by locating frequently occurring peaks. This was achieved by binning the $\mathrm{m} / \mathrm{z}$ values of the peaks from each fragmentation spectrum in $40 \mathrm{ppm}$ bins over a mass range of $50-2000$ Th and counting the frequency at which each bin occurred. ${ }^{9} \mathrm{We}$ also add the summed intensity of all fragments in a bin to determine the impact of the detected ion. From this inherently low resolution analysis the presence of diagnostic ions can be detected and subsequently used to enhance peptide identification algorithms to give additional confidence to peptide spectral matches (PSMs). In the case of phosphorylation on Tyrosine a diagnostic ion with a mass of $215.03474\left(\mathrm{C}_{8} \mathrm{H}_{10} \mathrm{O}_{4} \mathrm{NP}\right)$ is frequently generated in $\mathrm{HCD}$ experiments. Additionally, a set of immonium ions have been found to act as diagnostic indicators for phosphorylation of STY. A complementary approach of mining the spectral data is to locate frequently occurring mass differences. Here we implement this by looking for mass differences from the theoretical b- and $y$ series ions generated from the known peptide sequences. Also here, the mass differences are binned at the same resolution of $40 \mathrm{ppm}$. For the set of synthetic peptides we find that UVPD produces all of the frequent ions that HCD normally produces (Supplemental Figure 6a). However, in the UVPD spectra we find about 10 new frequent masses, which mostly represent immonium ions or other low mass ions associated to the amino acids present in the peptide sequence. The most dominant of these are immonium ions of the amino acids $\mathrm{N}, \mathrm{R}$ and $\mathrm{Q}$, which are indeed present in most of the peptides measured.

From the frequent mass difference analysis we reconfirm that UVPD fragmentation incurs a substantial lower percentage of modification specific losses, but also water losses are less readily formed which can improve peptide fragmentation for unmodified peptides as well (Supplemental Figure 5b). Further we find that the expected higher energy fragmentation pathways are very dominantly present in UVPD compared to HCD fragmentation; notably, $\mathrm{x}$ - and z-ion series display an increase of over $400 \%$. Surprisingly, we find evidence for ammonia-losses on a-ions for both fragmentation methods. Further there are no additional systematic mass differences, confirming

that UVPD is successfully accessing the higher energy fragmentation pathways without incurring further specific fragmentation, e.g., water losses. 

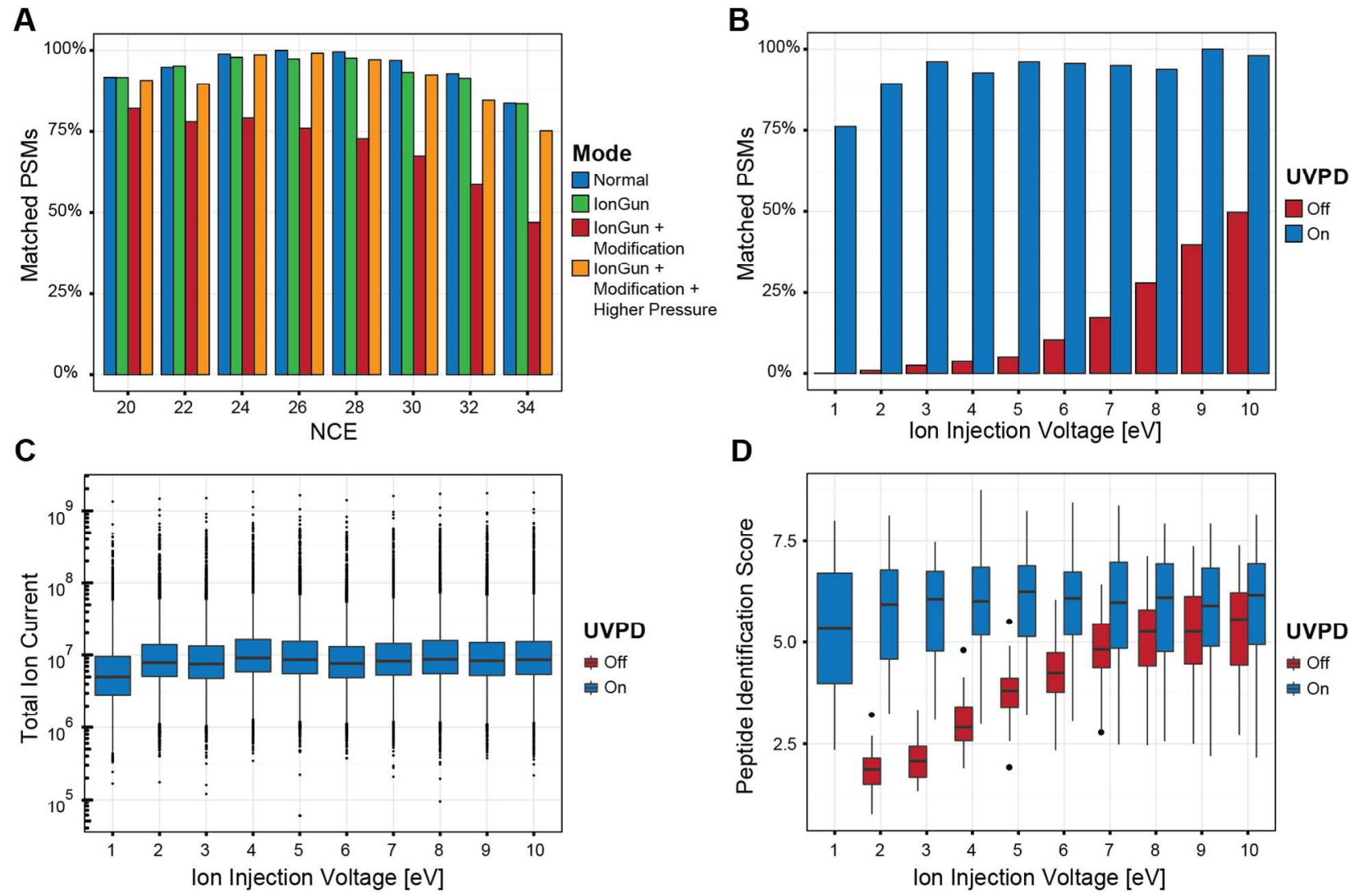

Supplemental Figure 1: Instrument performance at different modes of operation. A. Comparison of matched PSMs between normal operation and including the standard (normal) operation, "IonGun" triggering, after removal of the charge detector (IonGun + Modification), and increased gas pressure after removal of the charge detector (IonGun + Modification + Higher Pressure). B. UVPD identification rates at different ion injection voltages, producing supplemental activation. C. Transmission characteristics of the fragmentation cell expressed as total ion current as different ion injection voltages. D. With increasing collisional energy more informative fragment ions become available, increasing the peptide identification scores. The number of PSMs is normalized to the greatest number of PSMs identified. 


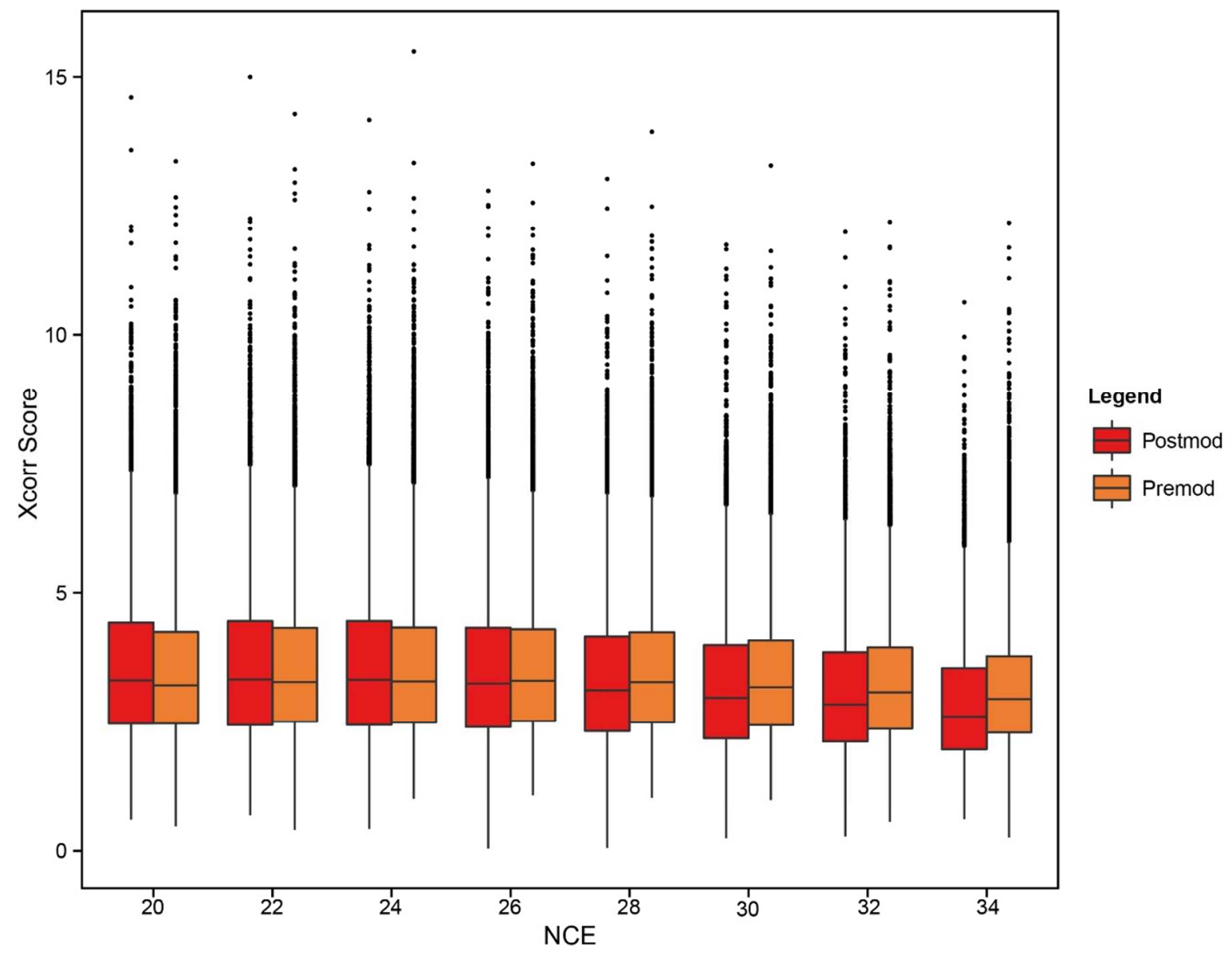

Supplemental Figure 2: Database scores for HeLa digest before and after modification at various NCE values 

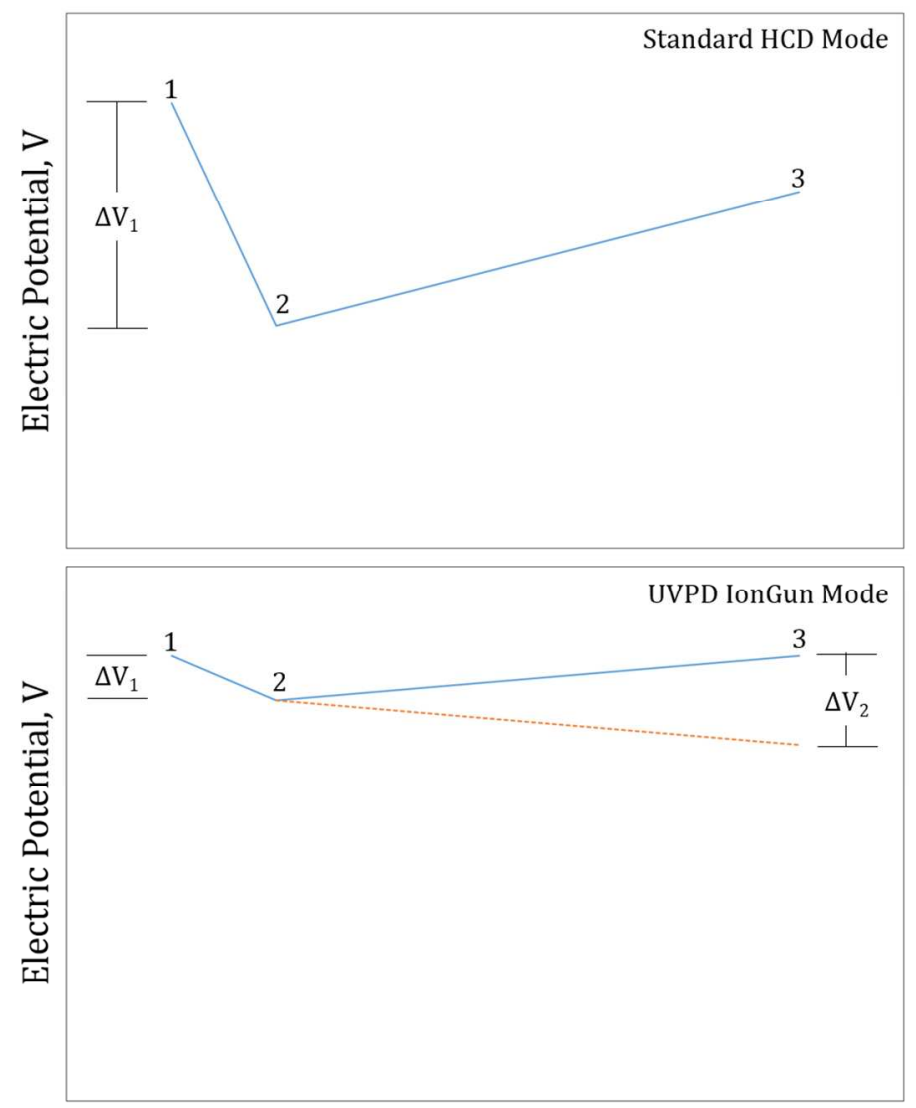

Axial Position

Supplemental Figure 3: Representation of electrical potentials used within HCD and UVPD mode of operation. The potential of the C-trap exit lens (1), the entrance to the HCD-cell (2), and the back of the HCD-cell (3) are shown. The fragmentation energy $\Delta V_{1}$ is defined by the NCE during standard HCD mode; however, during UVPD lonGun mode, this potential drop is diminished in magnitude to avoid activation of ions prior to irradiation. Once ions are injected into the HCD-cell, the electric potential is pulsed down (orange line) by a set voltage $\Delta \mathrm{V}_{2}$ in order to trigger the generation of the laser pulse. The potential depicted here are a general representation of voltages used and not reflective of precise voltages applied. 

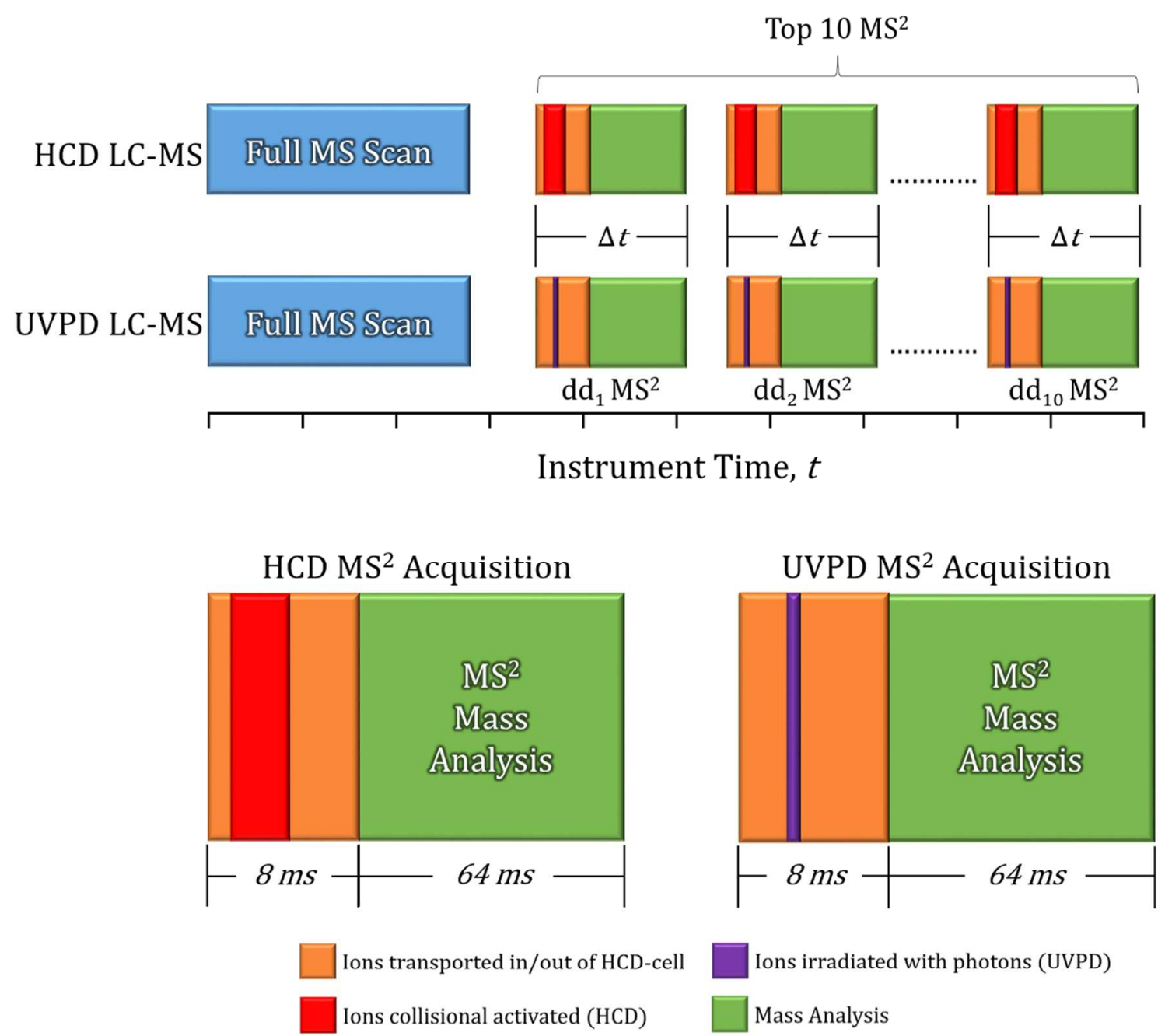

Supplemental Figure 4: Graphical depiction of the timescale of ion transport, activation, and mass analysis with both UVPD and HCD methods. For both HCD LC-MS and UVPD LC-MS (top), the Full MS Scan generates a list of the top $10 \mathrm{~m} / \mathrm{z}$ peaks that will be subjected to dd $\mathrm{MS}^{2}$. Upon completion of the Full scan, the ion of interest in transported into and out of the HCD-cell, which takes $8 \mathrm{~ms}$ (bottom). While the timescale of collisional activation during $\operatorname{HCD}(\sim \mu \mathrm{s}$ to $\mathrm{ms})$ is longer than the timescale of irradiation during UVPD (6 ns), the defined residence time within the HCD-cell $(8 \mathrm{~ms})$ prevents any duty cycle advantage for UVPD. 


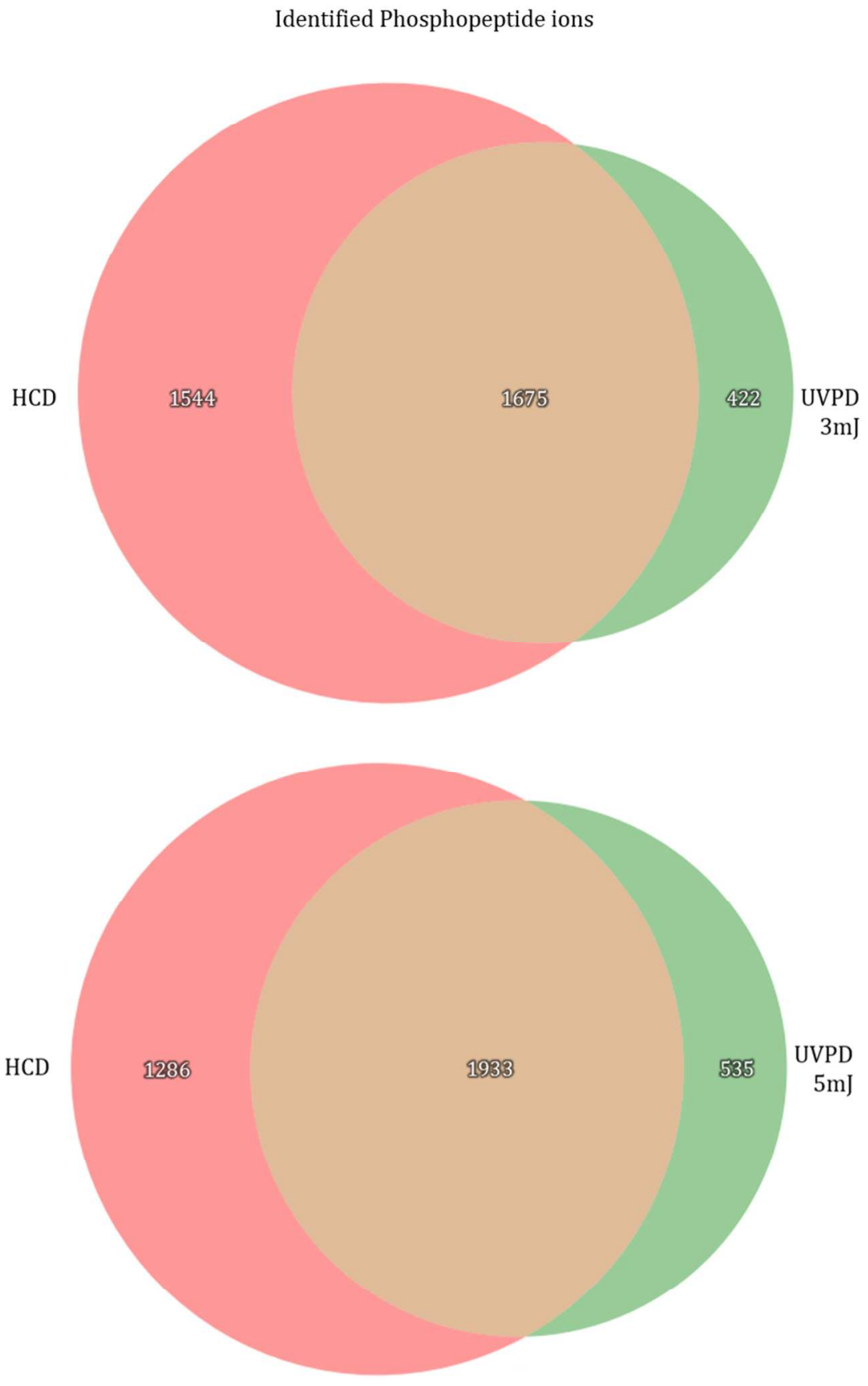

Supplemental Figure 5: Comparison of identified phosphopeptide ion with HCD and UVPD with $3 \mathrm{~mJ}$ (top) and $5 \mathrm{~mJ}$ (bottom) 

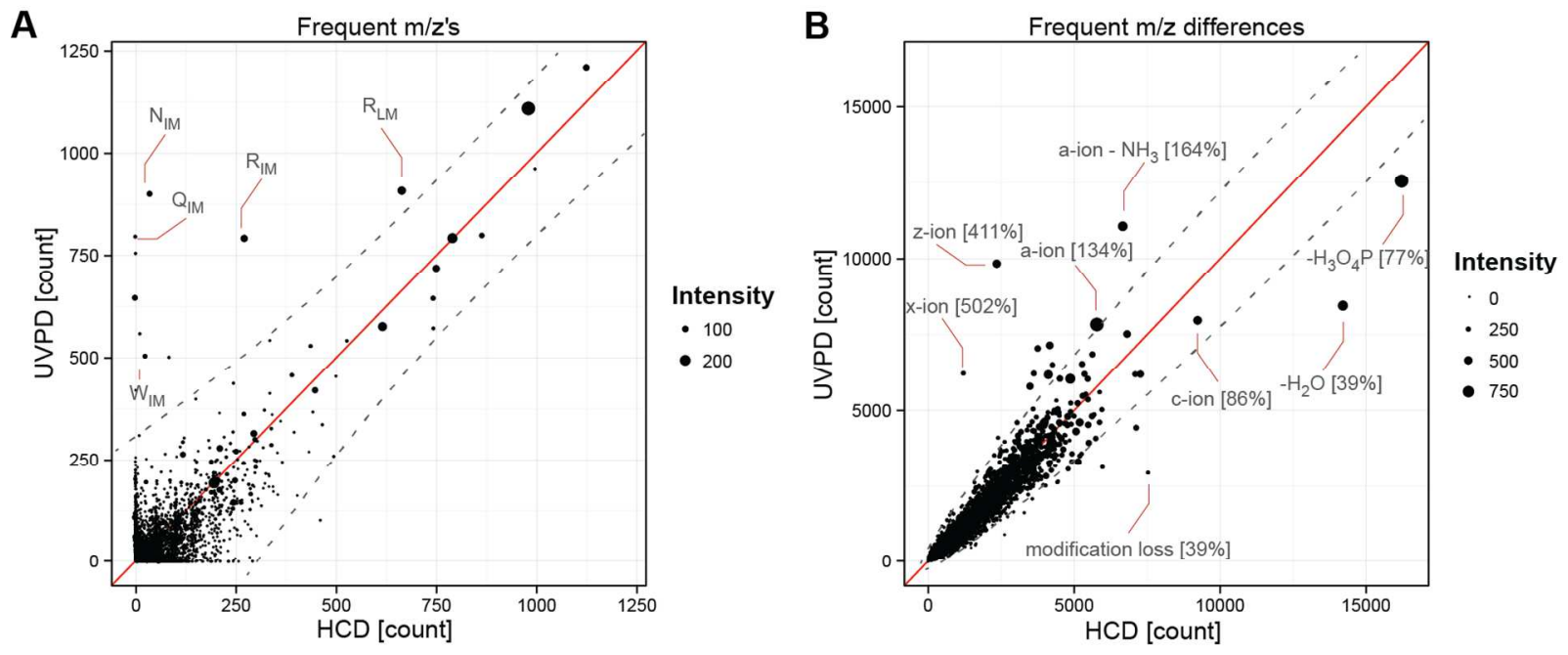

Supplemental Figure 6: Presence of UVPD specific fragments, where higher counts for a fragmentation method implies that that particular ion is more frequent for that method. A. Frequently detected $\mathrm{m} / \mathrm{z}$ values from UVPD and HCD spectra, after binning the spectra at $40 \mathrm{ppm}$. B. Frequently detected $\mathrm{m} / \mathrm{z}$ differences from UVPD and HCD spectra, binned at 20ppm precision. 


\begin{tabular}{|c|c|c|}
\hline \multicolumn{3}{|c|}{ HeLa LC Gradient } \\
\hline Time (min) & B\% & Flow $\left(\mathrm{nL} \mathrm{min}^{-1}\right)$ \\
\hline 0 & 6 & 250 \\
\hline 70 & 28 & 250 \\
\hline 73 & 100 & 250 \\
\hline 77 & 100 & 250 \\
\hline 80 & 4 & 250 \\
\hline 90 & 4 & 250 \\
\hline \multicolumn{3}{|c|}{ Phospho Enriched HeLa LC Gradient } \\
\hline Time (min) & $\mathrm{B} \%$ & Flow $\left(\mathrm{nL} \min ^{-1}\right)$ \\
\hline 0 & 6 & 250 \\
\hline 93 & 28 & 250 \\
\hline 97 & 100 & 250 \\
\hline 103 & 100 & 250 \\
\hline 107 & 4 & 250 \\
\hline 120 & 4 & 250 \\
\hline
\end{tabular}

Supplemental Table 1: LC gradients for the various samples analyzed by LC-MS 


\begin{tabular}{|c|c|c|c|c|c|c|}
\hline \multirow{3}{*}{$\begin{array}{l}\text { Phosphopeptide } \\
\text { Sequence }\end{array}$} & \multirow{3}{*}{ Phosphosite } & \multirow{3}{*}{$\begin{array}{l}\text { Charge } \\
\text { State }\end{array}$} & \multicolumn{4}{|c|}{ SEQUEST HT Xcorr Score } \\
\hline & & & \multicolumn{2}{|c|}{ All Ions } & \multicolumn{2}{|c|}{ b-/y-ions } \\
\hline & & & UVPD & HCD & UVPD & $\mathrm{HCD}$ \\
\hline NSVEQGRRL & S2 & {$[\mathrm{M}+3 \mathrm{H}]^{3+}$} & 2.79 & 2.32 & 2.10 & 2.01 \\
\hline APPDNLPSPGGSR & S12 & {$[\mathrm{M}+2 \mathrm{H}]^{2+}$} & 6.55 & 3.98 & 4.94 & 3.94 \\
\hline LIEDNEYTAR & Y7 & {$[\mathrm{M}+2 \mathrm{H}]^{2+}$} & 4.61 & 2.95 & 3.61 & 2.91 \\
\hline RSDGGHTVLHR & S2 & {$[\mathrm{M}+2 \mathrm{H}]^{2+}$} & 4.56 & 3.84 & 3.07 & 3.87 \\
\hline RSDGGHTVLHR & S2 & {$[\mathrm{M}+3 \mathrm{H}]^{3+}$} & 6.00 & 3.37 & 4.20 & 3.02 \\
\hline ENIMRSENSESQLTSK & S6 & {$[\mathrm{M}+2 \mathrm{H}]^{2+}$} & 5.21 & 3.82 & 2.84 & 3.76 \\
\hline ENIMRSENSESQLTSK & S6 & {$[\mathrm{M}+3 \mathrm{H}]^{3+}$} & 8.28 & 5.71 & 6.14 & 5.13 \\
\hline THILLFLPKSVSDYEGK & S10 & {$[\mathrm{M}+2 \mathrm{H}]^{2+}$} & 4.88 & 4.31 & 4.09 & 4.32 \\
\hline THILLFLPKSVSDYEGK & S10 & {$[\mathrm{M}+3 \mathrm{H}]^{3+}$} & 7.55 & 6.86 & - & 6.61 \\
\hline WWGSGPSGSGGSGGGK & S12 & {$[\mathrm{M}+2 \mathrm{H}]^{2+}$} & - & 5.28 & 5.19 & 5.06 \\
\hline LEKTIDDLEDK & $\mathrm{T} 4$ & {$[\mathrm{M}+2 \mathrm{H}]^{2+}$} & 4.06 & 4.60 & 2.83 & 4.28 \\
\hline RGDDSFGDK & S5 & {$[\mathrm{M}+2 \mathrm{H}]^{2+}$} & 4.31 & 3.15 & 3.34 & 2.66 \\
\hline LQLQGLDLSSR & S9 & {$[\mathrm{M}+2 \mathrm{H}]^{2+}$} & 4.67 & 3.56 & 3.77 & 3.22 \\
\hline TASDTDSSYAIPTAGMSPSR & Y9 & {$[\mathrm{M}+3 \mathrm{H}]^{3+}$} & 7.83 & - & 6.22 & - \\
\hline SVENLPEAGITHEQR & T11 & {$[\mathrm{M}+2 \mathrm{H}]^{2+}$} & 4.27 & 4.48 & 3.18 & 4.25 \\
\hline SVENLPEAGITHEQR & $\mathrm{T} 11$ & {$[\mathrm{M}+3 \mathrm{H}]^{3+}$} & 7.21 & 5.43 & 4.65 & 4.96 \\
\hline QLGEPEKSQDSSPVLSELK & S8, S16 & {$[\mathrm{M}+2 \mathrm{H}]^{2+}$} & 5.63 & 3.73 & 3.73 & 3.64 \\
\hline KFLSLASNPELLNLPSSVIK & S4, S17 & {$[\mathrm{M}+3 \mathrm{H}]^{3+}$} & 8.97 & 7.02 & - & 6.69 \\
\hline WWGSGPSGSGGSGGGK & S9, S12 & {$[\mathrm{M}+2 \mathrm{H}]^{2+}$} & 6.75 & 5.28 & 6.09 & 5.11 \\
\hline LQLQGLDLSSR & S9, S10 & {$[\mathrm{M}+2 \mathrm{H}]^{2+}$} & 4.26 & 4.11 & 3.58 & 3.74 \\
\hline WWGSGPSGSGGSGGGK & S7, S9, S12 & {$[\mathrm{M}+2 \mathrm{H}]^{2+}$} & 6.73 & 5.38 & 5.89 & 5.20 \\
\hline GSRAAKTLGSLGK & $\mathrm{S} 2, \mathrm{~T} 7, \mathrm{~S} 10$ & {$[\mathrm{M}+2 \mathrm{H}]^{2+}$} & 4.55 & 4.64 & 3.42 & 4.64 \\
\hline RYRVAKLGSSLGK & $\mathrm{Y} 2, \mathrm{~S} 9, \mathrm{~S} 10$ & {$[\mathrm{M}+2 \mathrm{H}]^{2+}$} & 3.35 & 3.70 & 2.31 & 3.54 \\
\hline SAGSRLTLSGR & S4, T7, S9 & {$[\mathrm{M}+2 \mathrm{H}]^{2+}$} & 3.90 & 3.27 & 3.13 & 3.30 \\
\hline FGSSNTDSAGALGTLR & $\begin{array}{c}\text { S3, S4, T14 } \\
\text { S4, S7, S9, }\end{array}$ & {$[\mathrm{M}+2 \mathrm{H}]^{2+}$} & 4.34 & 4.22 & 3.68 & 4.15 \\
\hline WWGSGPSGSGGSGGGK & $\begin{array}{c}\text { S12 } \\
\text { S2, S9, S10, }\end{array}$ & {$[\mathrm{M}+2 \mathrm{H}]^{2+}$} & 6.15 & 4.75 & 5.14 & 4.71 \\
\hline RSRVVKLGSSSLGK & S11 & {$[\mathrm{M}+2 \mathrm{H}]^{2+}$} & 2.73 & 4.88 & 1.90 & 4.29 \\
\hline \multicolumn{3}{|c|}{ Average } & 5.39 & 4.41 & 3.96 & 4.19 \\
\hline
\end{tabular}

Supplemental Table 2: Phospho peptide library. Table includes sequence, modification site, charge state, SEQUEST HT score with b-/y- ion search and all ion search. 
Figure
Associated Files

OR7_20150807_KLF_Hela_200ms_HCD_.raw

OR7_20150805_KLF_Hela_3mJ_200ms_raw

OR7_20150617_KLF_HeLa_NCE20.raw

OR7_20150617_KLF_HeLa_NCE22.raw

OR7_20150617_KLF_HeLa_NCE24.raw

OR7_20150617_KLF_HeLa_NCE26.raw

OR7_20150617_KLF_HeLa_NCE28.raw

OR7_20150617_KLF_HeLa_NCE30.raw

OR7_20150617_KLF_HeLa_NCE32.raw

OR7_20150617_KLF_HeLa_NCE34.raw

OR7_20150616_KLF_HeLa_iongun_100V_1ms_NCE20.raw

OR7_20150616_KLF_HeLa_iongun_100V_1ms_NCE22.raw OR7_20150616_KLF_HeLa_iongun_100V_1ms_NCE24.raw OR7_20150616_KLF_HeLa_iongun_100V_1ms_NCE26.raw OR7_20150616_KLF_HeLa_iongun_100V_1ms_NCE28.raw OR7_20150616_KLF_HeLa_iongun_100V_1ms_NCE30.raw OR7_20150616_KLF_HeLa_iongun_100V_1ms_NCE32.raw OR7_20150616_KLF_HeLa_iongun_100V_1ms_NCE34.raw OR7_20150622_KLF_HeLa_iongun_100V_1ms_NCE20_Post_Mod.raw OR7_20150622_KLF_HeLa_iongun_100V_1ms_NCE22_Post_Mod.raw OR7_20150622_KLF_HeLa_iongun_100V_1ms_NCE24_Post_Mod.raw OR7_20150622_KLF_HeLa_iongun_100V_1ms_NCE26_Post_Mod.raw OR7_20150622_KLF_HeLa_iongun_100V_1ms_NCE28_Post_Mod.raw OR7_20150622_KLF_HeLa_iongun_100V_1ms_NCE30_Post_Mod.raw OR7_20150622_KLF_HeLa_iongun_100V_1ms_NCE32_Post_Mod.raw OR7_20150622_KLF_HeLa_iongun_100V_1ms_NCE34_Post_Mod.raw OR7_20150904_KLF_Hela_NCE_20_P2_1.raw OR7_20150904_KLF_Hela_NCE_22_P2_1.raw OR7_20150904_KLF_Hela_NCE_24_P2_1.raw OR7_20150904_KLF_Hela_NCE_26_P2_1.raw OR7_20150904_KLF_Hela_NCE_28_P2_1.raw OR7_20150904_KLF_Hela_NCE_30_P2_1.raw OR7_20150904_KLF_Hela_NCE_32_P2_1.raw OR7_20150904_KLF_Hela_NCE_34_P2_1.raw

OR7_20150624_KLF_HeLa_iongun_100V_1ms_NCE10_Post_Mod_1_3mJ_UVPD.raw OR7_20150624_KLF_HeLa_iongun_100V_1ms_NCE10_Post_Mod_2_3mJ_UVPD.raw OR7_20150624_KLF_HeLa_iongun_100V_1ms_NCE10_Post_Mod_3_3mJ_UVPD.raw OR7_20150624_KLF_HeLa_iongun_100V_1ms_NCE10_Post_Mod_4_3mJ_UVPD.raw OR7_20150624_KLF_HeLa_iongun_100V_1ms_NCE10_Post_Mod_5_3mJ_UVPD.raw OR7_20150624_KLF_HeLa_iongun_100V_1ms_NCE10_Post_Mod_6_3mJ_UVPD.raw OR7_20150624_KLF_HeLa_iongun_100V_1ms_NCE10_Post_Mod_7_3mJ_UVPD.raw OR7_20150624_KLF_HeLa_iongun_100V_1ms_NCE10_Post_Mod_8_3mJ_UVPD.raw OR7_20150624_KLF_HeLa_iongun_100V_1ms_NCE10_Post_Mod_9_3mJ_UVPD.raw OR7_20150624_KLF_HeLa_iongun_100V_1ms_NCE10_Post_Mod_10_3mJ_UVPD.raw OR7_20150624_KLF_HeLa_iongun_100V_1ms_NCE10_Post_Mod_1_Control.raw OR7_20150624_KLF_HeLa_iongun_100V_1ms_NCE10_Post_Mod_2_Control.raw OR7_20150624_KLF_HeLa_iongun_100V_1ms_NCE10_Post_Mod_3_Control.raw OR7_20150624_KLF_HeLa_iongun_100V_1ms_NCE10_Post_Mod_4_Control.raw
Experimental Type

HCD; 25 NCE

UVPD; $3 \mathrm{~m}$

$\mathrm{HCD}$
Comments

HCD was performed on a tryptic digest of a HeLa lysate

UVPD was performed on a tryptic digest of a Hela lysate

HCD was performed prior to any modification HCD was performed
with "IonGun" triggering HCD was performe
with "lonGun"
triggering and
removal of the
charge detector HCD was performed
with "lonGun"
triggering and
removal of the
charge detector at a
higher gas flowrate
UVPD; $3 m$

Control
$B, D$ Ions were subjected to collision based fragmentation due to the translation energy from the various ion injection


HCD; 25 NCE

OR7_20150709_KLF_HeLa_iongun_100V_1ms_NCE25_Post_Mod_Trial_3_of_3.raw

OR7_20150708_KLF_HeLa_iongun_100V_1ms_NCE10_Post_Mod_2_3mJ_UVPD_Trial_1_of_3.raw

OR7_20150708_KLF_HeLa_iongun_100V_1ms_NCE10_Post_Mod_2_3mJ_UVPD_Trial_2_of_3.raw

OR7_20150708_KLF_HeLa_iongun_100V_1ms_NCE10_Post_Mod_2_3mJ_UVPD_Trial_3_of_3.raw

$$
\text { OR7_20150807_KLF_Hela_25ms_HCD_.raw }
$$

OR7_20150807_KLF_Hela_50ms_HCD_.raw

OR7_20150807_KLF_Hela_75ms_HCD_.raw

OR7_20150807_KLF_Hela_100ms_HCD_.raw

OR7_20150807_KLF_Hela_125ms_HCD_.raw

OR7_20150807_KLF_Hela_150ms_HCD_.raw

OR7_20150807_KLF_Hela_175ms_HCD_.raw

OR7_20150807_KLF_Hela_200ms_HCD_.raw

OR7_20150807_KLF_Hela_250ms_HCD_.raw

OR7_20150807_KLF_Hela_300ms_HCD_.raw

OR7_20150807_KLF_Hela_400ms_HCD_.raw

OR7_20150807_KLF_Hela_500ms_HCD_.raw

OR7_20150807_KLF_Hela_1000ms_HCD_.raw

OR7_20150805_KLF_Hela_3mJ_25ms_.raw

OR7_20150805_KLF_Hela_3mJ_50ms_.raw

OR7_20150805_KLF_Hela_3mJ_75ms_.raw

OR7_20150805_KLF_Hela_3mJ_100ms_.raw

OR7_20150805_KLF_Hela_3mJ_125ms_.raw

OR7_20150805_KLF_Hela_3mJ_150ms_raw

OR7_20150805_KLF_Hela_3mJ_175ms_.raw

OR7_20150805_KLF_Hela_3mJ_200ms_raw

OR7_20150805_KLF_Hela_3mJ_250ms_raw

OR7_20150805_KLF_Hela_3mJ_300ms_raw

OR7_20150805_KLF_Hela_3mJ_400ms_raw

OR7_20150805_KLF_Hela_3mJ_500ms_.raw

OR7_20150805_KLF_Hela_3mJ_1000ms_.raw

2015-03-18_Peptide_12_NCE25.RAW

2015-03-18_Peptide_12_100 Hz_5mJ.RAW

2015-03-21_Peptide_11_STATIC_3+_HCD_25.RAW

2015-03-21_Peptide_12_STATIC_2+_HCD_25.RAW

2015-03-21_Peptide_13_STATIC_2+_HCD_25.RAW

$\begin{array}{cc}\text { ACD; } 25 \text { NCE } & \\ \text { UVPD; 3mJ A,B,C } & \begin{array}{c}\text { Triplicate of HCD on } \\ \text { a tryptic HeLa digest }\end{array} \\ & \begin{array}{c}\text { Triplicate of UVPD } \\ \text { on a tryptic HeLa } \\ \text { digest }\end{array}\end{array}$

HCD; 25 NCE

D

UVPD; $3 \mathrm{~mJ}$

HCD; 25 NCE

$A, B, C, D, E$

HCD analysis of a phospho enriched HeLa digest

UVPD; $3 \mathrm{~mJ}$

$A, B, C, D, E$

UVPD (3mJ) analysis of a phospho enriched HeLa digest

UVPD; $5 \mathrm{~mJ}$

$A, B, C, D, E$

UVPD (5m) analysis

$$
\text { of a phospho }
$$
enriched HeLa digest HCD analysis of a singly

phosphorylated

peptide at 140,000

HCD; 25 NCE

mass resolution

UVPD analysis of a

singly

phosphorylated

peptide at 140,000

UVPD; $5 \mathrm{~mJ}$

HCD; 25 NCE

$B$

mass resolution

HCD analysis of a

phospho peptide

library via direct infusion 


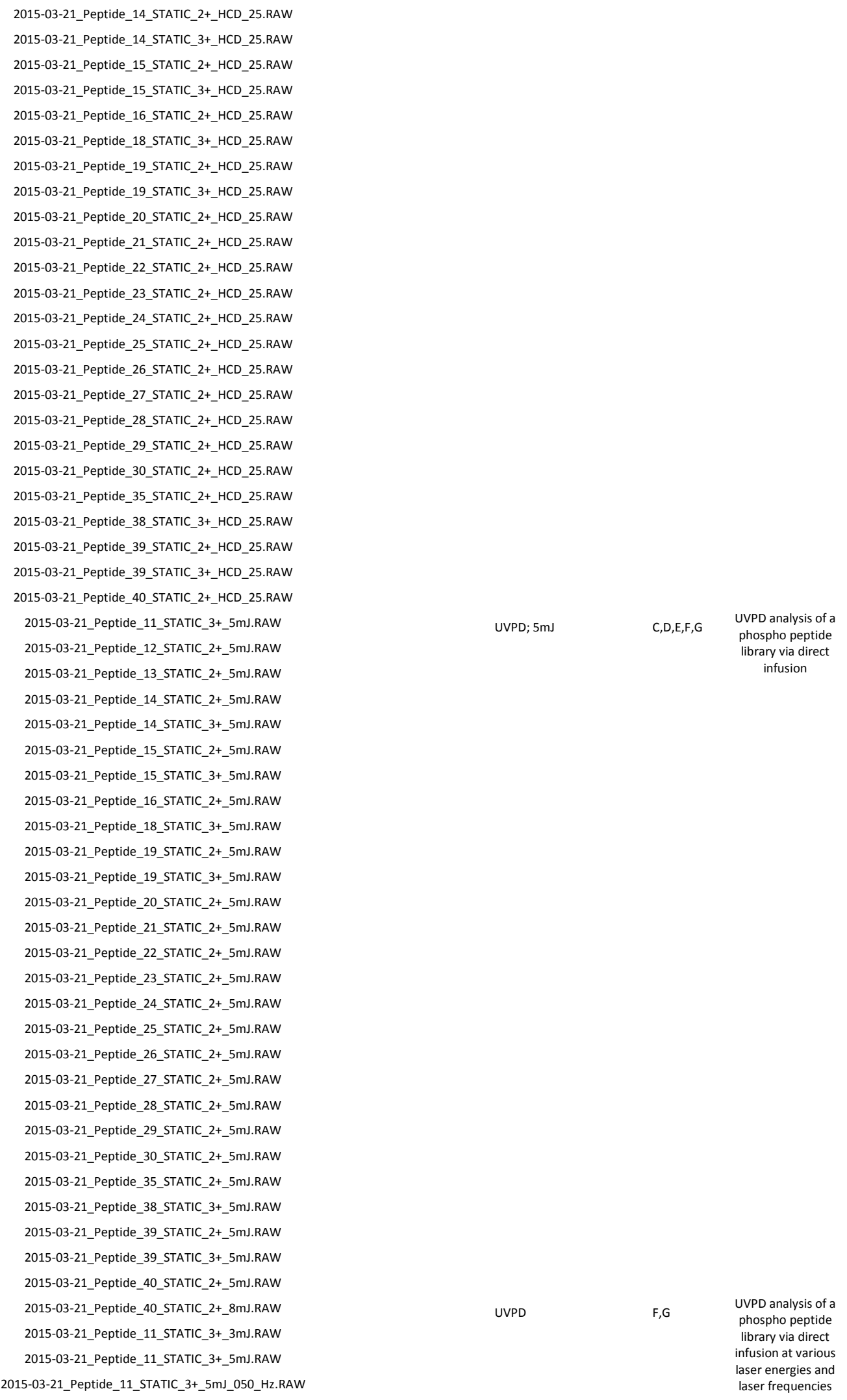

2015-03-21_Peptide_11_STATIC_3+ 5mJ100_Hz.RAW 2015-03-21_Peptide_11_STATIC_3+_5mJ_200_Hz.RAW 


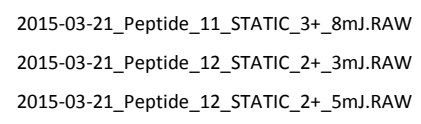


2015-03-21_Peptide_19_STATIC_3+_5mJ_050_Hz.RAW 2015-03-21_Peptide_19_STATIC_3+_5mJ_100_Hz.RAW 2015-03-21_Peptide_19_STATIC_3+_5mJ_200_Hz.RAW 2015-03-21_Peptide_19_STATIC_3+_8mJ.RAW 2015-03-21_Peptide_20_STATIC_2+_3mJ.RAW 2015-03-21_Peptide_20_STATIC_2+_5mJ.RAW 2015-03-21_Peptide_20_STATIC_2+_5mJ_050_Hz.RAW 2015-03-21_Peptide_20_STATIC_2+_5mJ_100_Hz.RAW 2015-03-21_Peptide_20_STATIC_2+_5mJ_200_Hz.RAW 2015-03-21_Peptide_20_STATIC_2+_8mJ.RAW 2015-03-21_Peptide_21_STATIC_2+_3mJ.RAW 2015-03-21_Peptide_21_STATIC_2+_5mJ.RAW 2015-03-21_Peptide_21_STATIC_2+_5mJ_050_Hz.RAW 2015-03-21_Peptide_21_STATIC_2+_5mJ_100_Hz.RAW 2015-03-21_Peptide_21_STATIC_2+_5mJ_200_Hz.RAW 2015-03-21_Peptide_21_STATIC_2+_8mJ.RAW 2015-03-21_Peptide_22_STATIC_2+_3mJ.RAW 2015-03-21_Peptide_22_STATIC_2+_5mJ.RAW 2015-03-21_Peptide_22_STATIC_2+_5mJ_050_Hz.RAW 2015-03-21_Peptide_22_STATIC_2+_5mJ_100_Hz.RAW 2015-03-21_Peptide_22_STATIC_2+_5mJ_200_Hz.RAW 2015-03-21_Peptide_22_STATIC_2+_8mJ.RAW 2015-03-21_Peptide_23_STATIC_2+_3mJ.RAW 2015-03-21_Peptide_23_STATIC_2+_5mJ.RAW 2015-03-21_Peptide_23_STATIC_2+_5mJ_050_Hz.RAW 2015-03-21_Peptide_23_STATIC_2+_5mJ_100_Hz.RAW 2015-03-21_Peptide_23_STATIC_2+_5mJ_200_Hz.RAW 2015-03-21_Peptide_23_STATIC_2+_8mJ.RAW 2015-03-21_Peptide_24_STATIC_2+_3mJ.RAW 2015-03-21_Peptide_24_STATIC_2+_5mJ.RAW 2015-03-21_Peptide_24_STATIC_2+_5mJ_050_Hz.RAW 2015-03-21_Peptide_24_STATIC_2+_5mJ_100_Hz.RAW 2015-03-21_Peptide_24_STATIC_2+_5mJ_200_Hz.RAW 2015-03-21_Peptide_24_STATIC_2+_8mJ.RAW 2015-03-21_Peptide_25_STATIC_2+_3mJ.RAW 2015-03-21_Peptide_25_STATIC_2+_5mJ.RAW 2015-03-21_Peptide_25_STATIC_2+_5mJ_050_Hz.RAW 2015-03-21_Peptide_25_STATIC_2+_5mJ_100_Hz.RAW 2015-03-21_Peptide_25_STATIC_2+_5mJ_200_Hz.RAW 2015-03-21_Peptide_25_STATIC_2+_8mJ.RAW 2015-03-21_Peptide_26_STATIC_2+_3mJ.RAW 2015-03-21_Peptide_26_STATIC_2+_5mJ.RAW 2015-03-21_Peptide_26_STATIC_2+_5mJ_050_Hz.RAW 2015-03-21_Peptide_26_STATIC_2+_5mJ_100_Hz.RAW 2015-03-21_Peptide_26_STATIC_2+_5mJ_200_Hz.RAW 2015-03-21_Peptide_26_STATIC_2+_8mJ.RAW 2015-03-21_Peptide_27_STATIC_2+_3mJ.RAW 2015-03-21_Peptide_27_STATIC_2+_5mJ.RAW 2015-03-21_Peptide_27_STATIC_2+_5mJ_050_Hz.RAW 2015-03-21_Peptide_27_STATIC_2+_5mJ_100_Hz.RAW 2015-03-21_Peptide_27_STATIC_2+_5mJ_200_Hz.RAW 2015-03-21_Peptide_27_STATIC_2+_8mJ.RAW 2015-03-21_Peptide_28_STATIC_2+_3mJ.RAW 2015-03-21_Peptide_28_STATIC_2+_5mJ.RAW 2015-03-21_Peptide_28_STATIC_2+_5mJ_050_Hz.RAW 2015-03-21_Peptide_28_STATIC_2+_5mJ_100_Hz.RAW 2015-03-21_Peptide_28_STATIC_2+_5mJ_200_Hz.RAW 


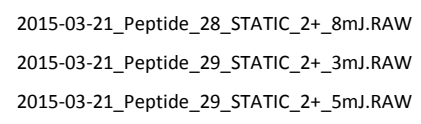




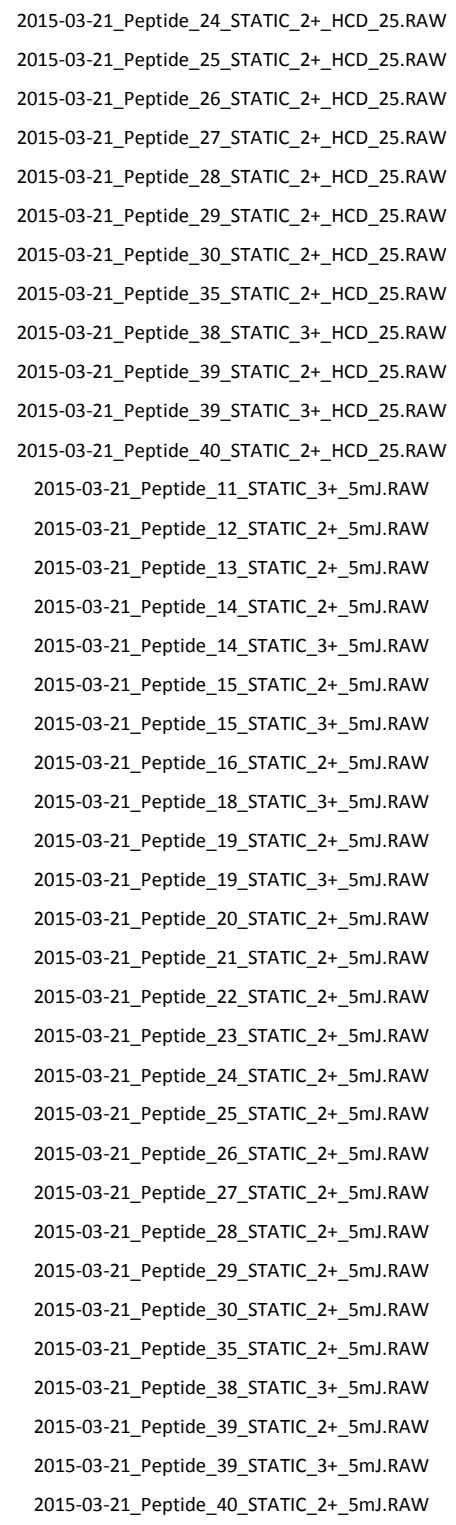

\section{Supplemental Table 3: Raw files correlated to Figures.}




\section{References:}

(1) Steen, H.; Mann, M. Nat Rev Mol Cell Biol 2004, 5, 699-711.

(2) Klammer, A. A.; Park, C. Y.; Noble, W. S. J. Proteome Res. 2009, 8, 2106-2113.

(3) Kim, T. Y.; Reilly, J. P. J. Am. Soc. Mass Spectrom. 2009, 20, 2334-2341.

(4) Thompson, M. S.; Cui, W. D.; Reilly, J. P. J. Am. Soc. Mass Spectrom. 2007, 18, 1439-1452.

(5) Morgan, J. W.; Russell, D. H. J. Am. Soc. Mass Spectrom. 2006, 17, 721-729.

(6) Taus, T.; Kocher, T.; Pichler, P.; Paschke, C.; Schmidt, A.; Henrich, C.; Mechtler, K. J Proteome Res 2011, 10, 5354-5362.

(7) Ihaka, R.; Gentleman, R. Journal of Computational and Graphical Statics 1996, 5, 299-314.

(8) Wickham, H. ggplot2; Springer-Verlag New York, 2009, p 213.

(9) Kelstrup, C. D.; Frese, C.; Heck, A. J. R.; Olsen, J. V.; Nielsen, M. L. Mol. Cell. Proteomics 2014, 13, 1914-1924. 